\title{
Radiation Damage Effects in Polarized Deuterated Ammonia
}

\author{
Paul M. McKee ${ }^{\dagger}$ \\ University of Virginia \\ for the E93-026 Collaboration
}

\begin{abstract}
Solid polarized targets utilizing deuterated ammonia, ${ }^{15} \mathrm{ND}_{3}$, offer an attractive combination of high polarization, high dilution factor and high resistance to polarization losses from radiation damage. Jefferson Laboratory Experiment E93-026 used ${ }^{15} \mathrm{ND}_{3}$ as a target material in a five-month form factor measurement, allowing a detailed study of it's performance. The dependence of the deuteron polarization on received dose by the ammonia and the effectiveness of annealing the material to recover performance lost to radiation damage will be discussed.
\end{abstract}

\section{INTRODUCTION}

A large number of high energy physics experiments of interest today require control of the spin degrees of freedom of the target and/or the beam. In such experiments the accuracy of the measurement (or, equivalently, the running time of the experiment) depends strongly on the degree of polarization attained, thus it is important to be able to characterize the polarization performance of the target used.

This article discusses the performance of a solid polarized target using deuterated ammonia, ${ }^{15} \mathrm{ND}_{3}$. This target was operated by the University of Virginia Polarized Target Group for experiment E93-026, a measurement of the electric form factor of the neutron, $G_{E}^{n}$, which ran in late 2001 in Hall C of Jefferson Laboratory, Newport News, Virginia. The focus will be on patterns observed in the polarization performance of the material as a function of deposited beam charge, and the techniques used to maximize this performance.

\section{POLARIZED TARGET OVERVIEW}

The target uses the technique of Dynamic Nuclear Polarization (DNP) to enhance the polarization of the spin species in the material. In DNP, the material is first doped to create paramagnetic centers which couple to the nuclei to be polarized. In the case of ammonia, this doping is accomplished by placing it in a beam of ionizing radiation.

The material is then placed in a high, uniform magnetic field, cooled to $1 \mathrm{~K}$ or less, and irradiated with microwave energy designed to drive transitions in the coupled-spin system. Using this technique, a system running at $5 \mathrm{~T}$ and $1 \mathrm{~K}$ can produce deuteron polarizations above $40 \%$. The polarization typically takes a portion of an hour to reach its 
maximum value, at which point the beam may be put on the target and the experimental measurement begun.

The beam has both a prompt and a cumulative effect on the target polarization. First, since the beam adds a small amount of heat to the material, it reduces the efficiency of the polarizing process, resulting in a polarization loss of 2 or $3 \%$ (absolute) over a period of about a minute. Over time, the beam also damages the material, producing additional paramagnetic centers that can allow some of the spins to relax, reducing the overall polarization of the target. In deuterated ammonia it is thought that the principle source of this relaxation is the production of atomic deuterium in the material. A side effect is that the microwave frequency for optimum polarization slowly changes with dose and must be tracked by the target operator. As the radiation damage of the material continues, the target polarization continues to decrease until a point at which it is no longer practical to run the experiment.

The performance of the damaged material may be largely restored by annealing the target. This involves shutting off the beam and the microwaves and warming the material for a length of time. The optimal temperature and duration depend on the history of the material, but range from 70 to $115 \mathrm{~K}$ and 10 to 60 minutes. After the anneal, the procedure is similar to that for new material: the target is cooled, the microwaves restored (at their original frequency) and the polarization builds to a maximum value, at which point beam may be reintroduced.

\section{MATERIAL PREPARATION}

As mentioned in the previous section, the deuterated ammonia is prepared by doping it with ionizing radiation. This may be done with the material in a special preparation dewar at $87 \mathrm{~K}$ ("warm irradiation") or in the actual polarized target at $1 \mathrm{~K}$ ("cold irradiation"). Irradiation produces the paramagnetic centers needed for the DNP process, and also changes the color of the icy material from a milky white to a deep purple.

Warm-irradiated material may undergo an additional step, known as tempering, in which the material is held above a liquid nitrogen bath until the purple color fades to a dirty white color. For experiment E93-026, two batches of cold-irradiated material and four batches of warm-irradiated, tempered material were used. A seventh batch consisted of a mixture of cold-irradiated and warm/tempered material.

\section{PERFORMANCE}

Experiment E93-026 was unique in that it put more beam on a deuterated ammonia target than any previous experiment. In all, $350 \mathrm{mC}$ was deposited on the various batches of deuterated ammonia. This allowed the observation of long-term behavior difficult to isolate in shorter experiments, and further exploration of previously known effects. 


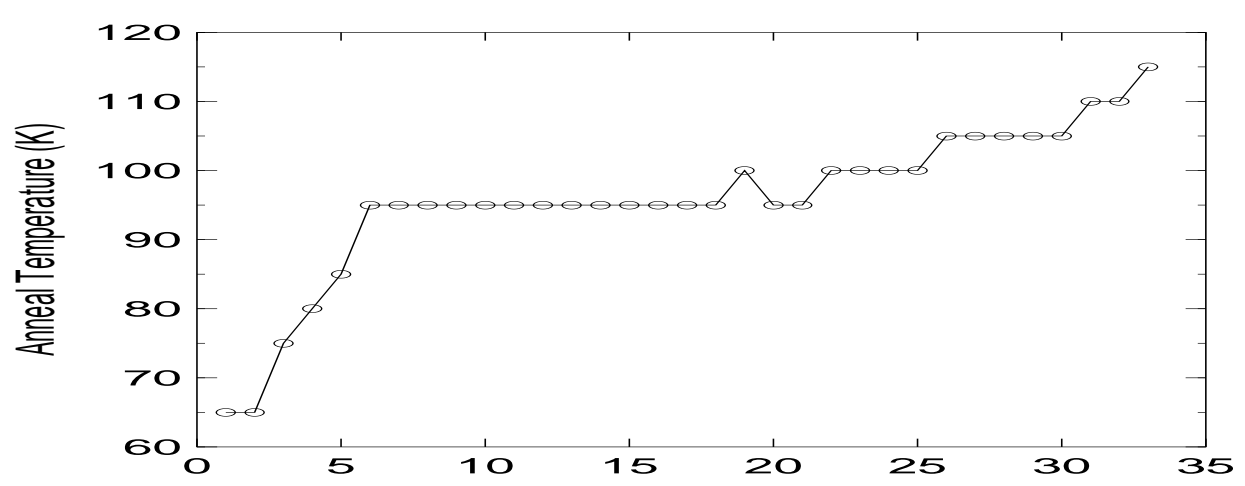

FIGURE 1. Temperatures used in anneal cycles on one load of material.

\section{Anneal Temperature}

The degree to which an anneal restores the performance of the material depends on both the anneal temperature and the length of time spent at that temperature. Many time/temperature combinations were tried during E93-026 and the degree of restoration noted for each.

The basic pattern that emerged is that for the first few anneal cycles, relatively cold and short anneals ( 70 to $80 \mathrm{~K}$ and 10 to 20 minutes) produce satisfactory restoration. After that, for perhaps the next 15 cycles, the best performance comes with $95 \mathrm{~K} / 60$ minute anneals. Eventually, increases in temperature up to as high as $115 \mathrm{~K}$ become necessary, although there is no evidence that anneals longer than 60 minutes are needed. Figure 1 shows the history of temperatures used for a warm-irradiated, tempered batch of material.

In judging whether a given anneal is successful it is important not to focus solely on the maximum polarization achieved after the anneal, because often deuterated ammonia will polarize higher after an anneal than in the previous cycle even if the anneal conditions were not optimal. This effect is most pronounced during the first several cycles of the material's life.

A more reliable method is to observe the microwave frequency that produces the maximum polarization. As mentioned above, the optimum frequency slowly drifts as radiation damage in the material accumulates (to lower frequencies for positive enhancement and to higher frequencies for negative enhancement). If the conditions of the anneal were well suited to the history of the material, the frequency will usually return to the value it had at the beginning of the previous anneal cycle.

\section{Rate of Polarization Decay}

In ${ }^{15} \mathrm{NH}_{3}$, a pattern emerges in which the rate of polarization decay increases with each anneal cycle. Although the maximum polarization may not decline much, the 


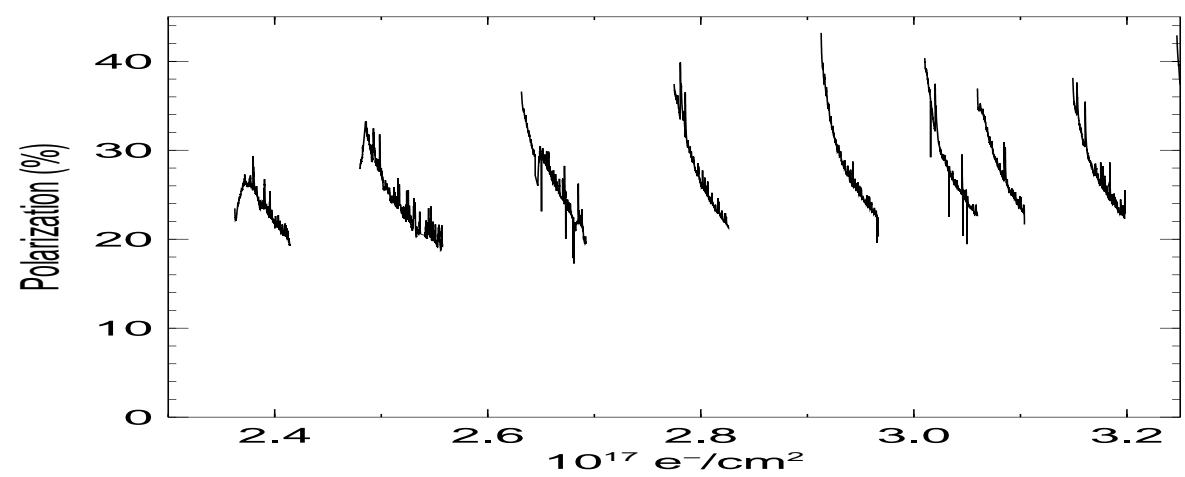

FIGURE 2. Evidence of slight increase in decay rate with dose.

decrease in running efficiency caused by the faster decays and more frequent anneals eventually requires the material to be replaced with a fresh sample.

In most experiments, this effect is not seen with deuterated ammonia. In E93-026 there is evidence of a somewhat faster decay rate, though nothing near the rate increase in $\mathrm{NH}_{3}$, which can be more than a factor of two. An example of this effect in deuterated ammonia is shown in Figure 2.

\section{Irradiation Temperature}

The more common method of irradiating ammonia is to use a separate irradiation dewar, often at a different lab than the one at which the experiment will take place, filled with liquid Argon to produce an $87 \mathrm{~K}$ bath. We call this warm irradiation.

Cold-irradiation is done in the polarized target apparatus itself, with the refrigerator running at 1 to $1.5 \mathrm{~K}$. It is often more convenient because beam time that does not require a polarized target, such as commissioning or calibration periods, can be used to prepare target material.

There is a noticeable difference in performance between warm- and cold-irradiated material. Warm-irradiated material polarizes more quickly, polarizes higher, and experiences more gradual radiation damage than cold-irradiated material. Figure 3 shows the performance of both types of material.

\section{Material Tempering}

The process of tempering the material before use in the target shows promise. The technique was first attempted during a test run of the target with old, poorly performing material that had been used in a past experiment. After tempering, it polarized to $50 \%$.

For E93-026, all four warm-irradiated batches of material were tempered before use. Since the performance of tempered, cold-irradiated material is unknown, it would not 


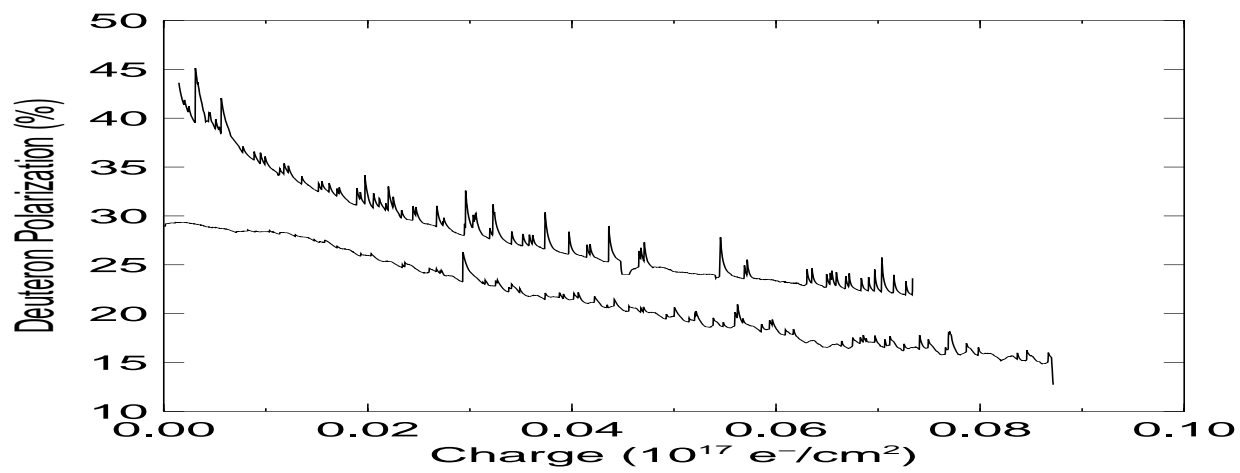

FIGURE 3. Characteristic performance of cold-irradiated (lower plot) and warm-irradiated (upper plot) material.

have been appropriate to use it for the first time during an experiment. Future tests will investigate the performance of this type of material.

\section{CONCLUSION}

Experiment E93-026 provided an opportunity to study the behavior of deuterated ammonia under accumulated doses larger than in any previous experiment. In analyzing the over 370,000 polarization measurements, several interesting aspects of the material's performance emerged.

First, the optimal annealing conditions appear to be $95 \mathrm{~K}$ for 60 minutes for all but the first few and last several cycles of a material's life. Second, it appears that the polarization decay rate does increase after several anneal cycles, but only slightly. Third, the performance of material irradiated at $87 \mathrm{~K}$ is better in several ways than material irradiated at $1 \mathrm{~K}$. Finally, tempering a load of material by warming it slowly until the purple color disappears results in higher polarizations and faster polarization buildups.

Taken together, these new observations indicate that although deuterated ammonia has a long history of success as a polarized target material, further study provides information useful for improving its performance in future experiments.

\section{ACKNOWLEDGMENTS}

The author would like to thank D. G. Crabb for many helpful discussions. This work was supported by Department of Energy contract DE-FG05-86ER40261, and by the Institute of Nuclear and Particle Physics of the University of Virginia. 